\title{
Lung cancer with dermatomyositis as the initial diagnosis: A case report
}

\author{
TIANTIAN ZHANG ${ }^{1}$, QIONG WU ${ }^{1}$, SHUKUI QIN ${ }^{2}$, ZISHU WANG $^{1}$, YAWEI LI $^{1}$, RUI WANG ${ }^{1}$ and FANG SU ${ }^{1}$ \\ ${ }^{1}$ Department of Medical Oncology, The First Affiliated Hospital of Bengbu Medical College, Bengbu, Anhui 233004; \\ ${ }^{2}$ Department of Medical Oncology, PLA Cancer Center, Nanjing Bayi Hospital, Nanjing, Jiangsu 210002, P.R. China
}

Received November 2, 2018; Accepted March 21, 2019

DOI: $10.3892 / \operatorname{mco} .2019 .1851$

\begin{abstract}
It is well-known that dermatomyositis (DM) is associated with a variety of malignancies, and the cancer-specific mortality rate is high among patients who develop paraneoplastic DM. Paraneoplastic syndromes are described in $\sim 10-25 \%$ of adult patients with DM. Although paraneoplastic syndromes are associated with various malignancies, the most common is lung cancer, with different histological types of lung cancer being associated with different paraneoplastic syndromes. We herein report the case of a patient with lung cancer and DM, aiming to provide a useful guide to diagnosis and treatment by improving the understanding of these diseases for clinicians. Early detection, accurate diagnosis and timely treatment are crucial for prolonging patient survival.
\end{abstract}

\section{Introduction}

Dermatomyositis (DM) represents an idiopathic inflammatory connective tissue disease, characterized by inflammation of the muscles and the skin. The skin manifestations include a heliotrope rash (blue-purple discoloration) of the upper eyelids, red patches on the face and upper trunk, Gottron's papules and a raised scaly eruption of the knuckles (1). The first case of DM associated with gastric cancer was reported by Stertz in 1916 (2). A number of studies have been conducted to investigate the association between DM and malignancy. Approximately $10-25 \%$ of DM cases are associated with various malignancies and are classed as paraneoplastic syndromes (1). We herein report the case of a patient with small-cell lung cancer accompanied by paraneoplastic DM.

Correspondence to: Dr Fang Su, Department of Medical Oncology, The First Affiliated Hospital of Bengbu Medical College, 287 Changhuai Road, Bengbu, Anhui 233004, P.R. China

E-mail: sufang2899@163.com

Key words: dermatomyositis, paraneoplastic syndromes, lung cancer

\section{Case report}

The patient was a 48-year-old woman without a history of smoking or drinking, who developed symmetrical erythema and numerous papules on the cheeks, chest and back, without an obvious inducing factor. The skin lesions were accompanied by intense pruritus. The patient was diagnosed with eczema at a local hospital, but no significant improvement was observed after antiallergic treatment with oral levcetirizine for 1 month. There was progressive chest tightness, asthma, heart palpitations and enlargement of the skin lesions. Extensive erythema was observed on the trunk and limbs. Skin erythema and papules were also observed on the dorsum of the hands and extensor surfaces of the knuckles. The patient also exhibited symmetrical progressive weakness of both upper and lower (muscle strength was assessed as level 4), characterized by difficulty in lifting the upper limb, a small angle $\left(<90^{\circ}\right)$ between the abducted arms and the trunk, difficulty in standing up after crouching, and symmetric involvement of the limbs (Fig. 1). In addition, the patient developed cough and aggravation of the chest congestion, asthma and dyspnea. The patient was admitted to the Department of Dermatology of the First Affiliated Hospital of Bengbu Medical College (Bengbu, China) on February 19, 2018. The results of electromyography (EMG) revealed normal motor nerve conduction velocity (MCV) and sensory nerve conduction velocity of the right median, bilateral ulnar, right peroneal and right tibial nerves. Positive sharp waves were observed in the left and right anterior tibialis muscles and the right deltoid muscle. Spontaneous potentials were observed when the muscles were at rest. The amplitude of the motor units was normal, but the duration was shortened. The conclusion of the MCV assessment was myogenic damages. A computed tomography (CT) scan of the chest showed a space-occupying lesion, bilateral pleural effusion and hydropericardium in the left lung (Fig. 2). Ultrasound-guided pericardial puncture and exfoliative cytological examination were performed. Malignant cells were identified, resembling a poorly differentiated carcinoma. Lung biopsy under ultrasonographic guidance was also performed and the results of the pathological examination revealed small-cell lung cancer (Fig. 3). The results of the laboratory examinations were as follows: Lactate dehydrogenase $915 \mathrm{U} / 1$ (reference 
value: $313-618 \mathrm{U} / 1)$, erythrocyte sedimentation rate (ESR) $34 \mathrm{~mm} / \mathrm{h}$ (reference value: $0-20 \mathrm{~mm} / \mathrm{h}$ ), creatine kinase (CK) $846 \mathrm{U} / 1$ (reference value: 40-200 U/1), CK MB isoenzyme, $31 \mathrm{U} / 1$ (reference value: 0-25 U/1) and blood calcium $2.0 \mathrm{mmol} / \mathrm{l}$ (reference value: $2.11-2.52 \mathrm{mmol} / \mathrm{l}$ ). Taking into consideration the patient's history, symptoms, signs and the results of the physical and laboratory examinations, the patient was diagnosed with small-cell lung cancer with bilateral pleural and pericardial effusion and DM (TxNxM1 stage IV). Glucocorticoids (80 $\mathrm{mg}$ ) were administered to treat DM for 5 days, and treatment with etoposide $100 \mathrm{mg}$ d1-5+ cisplatin $30 \mathrm{mg} \mathrm{d} 1-4$ (EP regimen) was initiated on March 2, 2018. After one course of chemotherapy, there were no obvious gastrointestinal adverse reactions or bone marrow suppression. The patient then received the second cycle of chemotherapy. However, on March 19, 2018, the patient became unconscious while hospitalized and her family members requested treatment discontinuation. The patient was discharged from the hospital and succumbed to respiratory failure on March 22, 2018.

\section{Discussion}

$\mathrm{DM}$ is an autoimmune disease characterized by inflammatory changes involving the muscle and skin, manifesting as proximal muscle weakness and characteristic skin rashes. The clinical manifestations of DM do not differ between patients with and those without concurrent malignancies (1). The incidence of DM is $\sim 1 / 100,000$ (3) and it may occur at any age, although its incidence is high among adults aged $>45$ years. DM in children is not associated with malignancies (1). The diagnosis of DM is mainly based on the diagnostic criteria proposed by Bohan and Peter in 1975 (3): i) Asymmetrical progressive muscle weakness in the lower and upper limbs, with or without dysphagia or dyspnea; ii) myositis confirmed by muscle biopsy; iii) increased serum creatine kinase levels; iv) abnormal EMG manifesting as primary muscle injury; v) characteristic skin lesions. Our patient was diagnosed with DM based of the abovementioned criteria (i and iii-v). Among all adult DM patients, 10-25\% develop DM as a paraneoplastic syndrome, which may be associated with various malignancies. The studies of De Souza and Shinjo (4) demonstrated that cancer prevalence may be as high as $8.6 \%$ among newly diagnosed DM patients, and the incidence of malignancies is the highest within the first year after the diagnosis of DM. DM-associated malignancies include breast, ovarian, lung and gastrointestinal cancers (5). The most common is adenocarcinoma, accounting for $\sim 70 \%$ of all tumors in $\mathrm{DM}$ and polymyositis (PM) patients (5). Combined with the diagnostic criteria confirming DM and the histopathological results of the lung biopsy, our patient was diagnosed with small-cell lung cancer with bilateral pleural and pericardial effusion and DM (TxNxM1 stage IV).

Paraneoplastic syndromes, which are caused by tumor-secreted substances, abnormal immune responses, or other unknown causes, can affect most systems and organs, including the endocrine, nervous, digestive and hematopoietic systems, joints, kidneys and skin, with corresponding clinical manifestations. These manifestations are not related to the invasion and metastasis of primary tumors, and usually result from tumor-produced functional polypeptides, hormones and cytokines, or by the immune cross-reaction between tumors and normal host tissues (6). Paraneoplastic syndromes are a group of non-metastatic systemic diseases associated with malignancies and they occur in $7-15 \%$ of cancer patients, affecting multiple organs of the entire body (7). In the majority of the cases, paraneoplastic syndromes are the first or most prominent manifestation of the underlying malignancy, and they may present prior to the diagnosis of the tumor with a mean interval of 2-12 months (8).

Paraneoplastic syndromes are associated with a variety of malignancies, the most common of which is lung cancer (9). Approximately $10 \%$ of lung cancer patients develop paraneoplastic syndromes, and different histological types of lung cancer are associated with different paraneoplastic syndromes (9). Humoral hypercalcemia of malignancy in squamous cell carcinoma and syndrome of inappropriate antidiuretic hormone secretion in small-cell lung cancer are the two most common types. The most common type of DM-related lung cancer is small-cell lung cancer (29\%), followed by squamous cell carcinoma $(21 \%)$ and adenocarcinoma (8\%) (5). The severity of the symptoms is not related to the size of the primary tumor and, in the majority of the cases, paraneoplastic syndromes are present before cancer diagnosis (7). The patient in the present case was diagnosed with small-cell lung cancer. Sparsa et al (10) studied 40 patients with DM and PM and found that the following clinical characteristics may be associated with malignancies: Systemic symptoms, no Raynaud's phenomenon, rapid onset of myositis, elevated ESR and CK levels. The systemic symptoms were obvious, the ESR and CK levels were both higher than normal and there was no Raynaud's phenomenon in our patient, consistently with the abovementioned criteria. Therefore, the patient was considered likely to have an underlying malignancy. Basset-Seguin et al (11) reported that the 3-year survival rate of DM patients was $57 \%$, while the 2-year survival rate of DM patients with cancer was $25 \%$. For most paraneoplastic syndromes, the best treatment is to treat the underlying malignancy. Glucocorticoids are the main drugs used in the treatment of DM, but other immunomodulatory treatments for paraneoplastic DM are also needed (12). The muscular symptoms can be improved with treatment targeting the tumor, but the skin involvement, which is often difficult to treat, may persist (13). Good treatment outcomes may be achieved by using corticosteroids and a variety of non-steroid drugs, including methotrexate, antimalarial drugs, mycophenolate, azathioprine and cyclosporine. Immunoglobulins, rituximab and tumor necrosis factor- $\alpha$ inhibitors are beneficial for patients with drug resistance $(13,14)$. For patients diagnosed with DM, additional evaluation is recommended, including whole-blood cell count, metabolic function examination, urine and hematological examination, chest and abdominal CT scan, among others. For female patients, additional examinations should include breast examination, pelvic CT scan and gynecological examination (5). The patient was misdiagnosed with only DM, since only skin manifestations were present at the early stages of the disease. No chest or abdominal CT examination was performed to rule out other systemic 


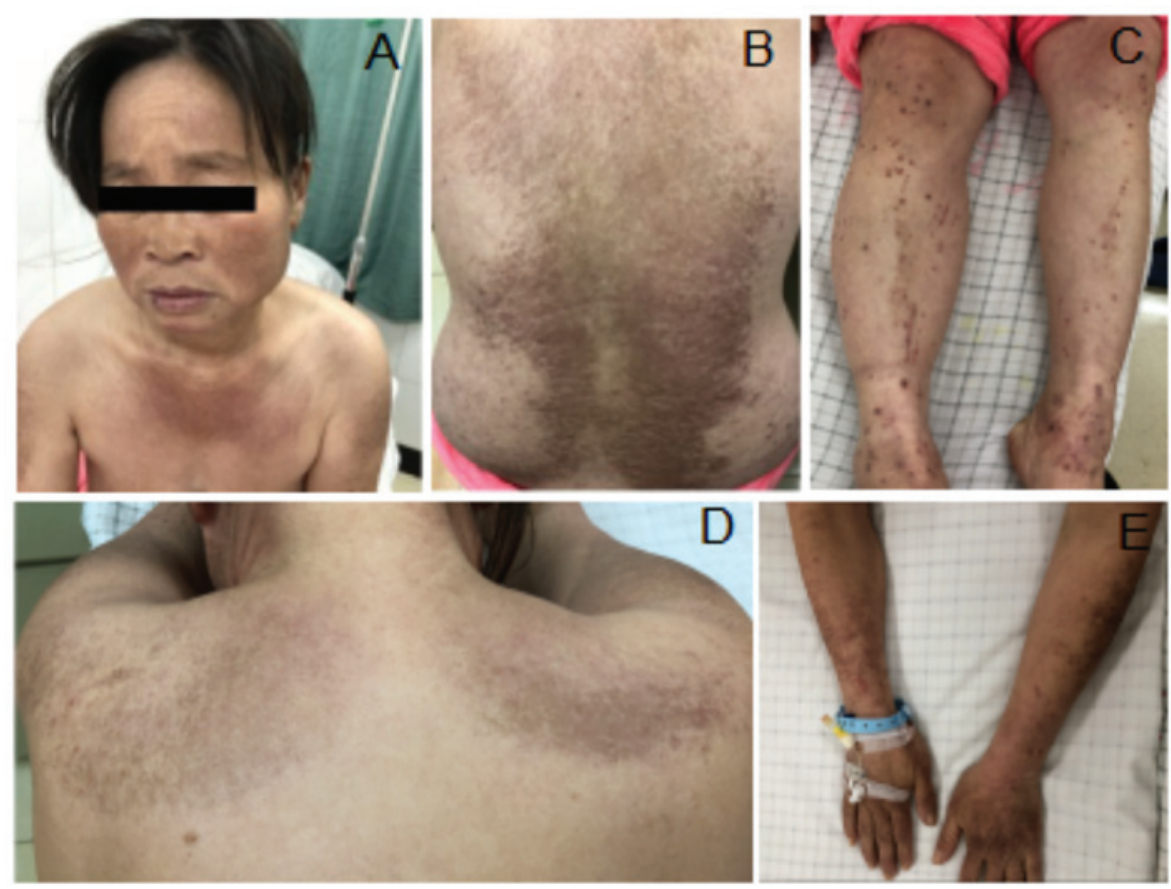

Figure 1. (A-E) On physical examination, the patient displayed skin lesions on the face, trunk and limbs. (A) Characteristic skin lesions ('V' sign) may be seen on the chest.

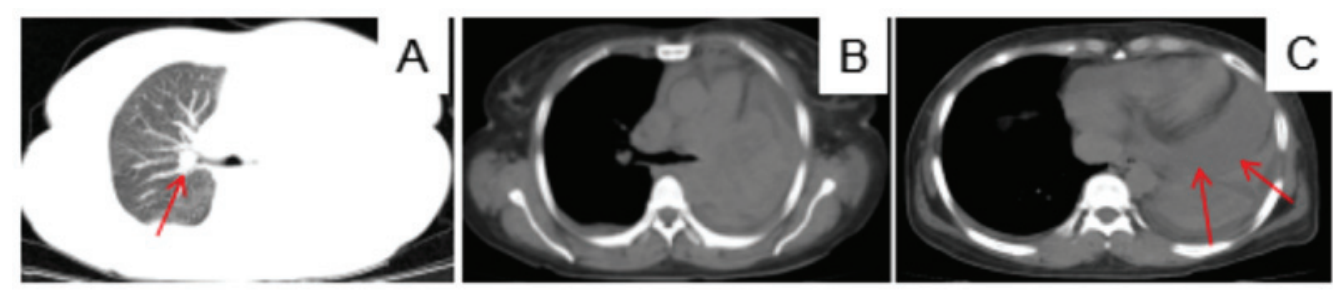

Figure 2. Plain computed tomography scan of the chest showing (A) an irregular soft tissue mass shadow at the hilum of the right lung (arrow); (B) left main bronchial occlusion with left atelectasis; (C) massive pericardial effusion (arrows).
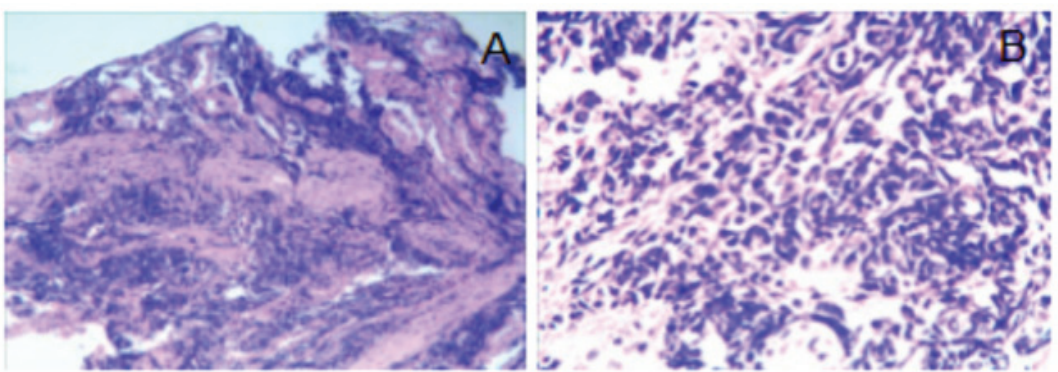

Figure 3. On histopathological examination of the lung tumor, the cells were small, round or spindle-shaped, with extrusion deformation and increased nuclear: Cytoplasmic ratio, consistent with the pathological findings of small-cell lung cancer (hematoxylin and eosin staining, magnification (A) x200 and (B) x400.

diseases. The patient only received treatment targeting DM at the local hospital; she developed chest congestion and palpitations when transferred to our hospital, which were considered to be the result of pericardial effusion. At that time, the patient had terminal cancer with distant metastasis. She succumbed to the disease within 1 month after being diagnosed with small-cell lung cancer.

The clinical characteristics of DM concurrently with lung cancer may be non-specific and easy to overlook. The patient presented only with skin manifestations at the early stages. As a result, lung cancer was discovered at a late stage. The treatment efficacy and prognosis are very poor. At present, the main challenge in treating cancer-associated PM or DM is timely identification of the underlying malignancies. Extensive examination should be performed in DM and PM patients to eliminate the possibility of malignancy, particularly for patients aged $>40$ years. This case stresses the fact that early diagnosis and timely treatment are crucial for patient prognosis, and 
the diagnosis of DM should always be considered as an early warning sign of malignancy.

\section{Acknowledgements}

Not applicable.

\section{Funding}

The present study was supported by funding from the National Natural Sciences Foundation of China (grant no. 81702450), the Natural Science Research major Project of Education Office of Anhui Province (grant no. KJ2016SD40) and Support Program for Outstanding Young Talents in Colleges and Universities of Anhui Province (grant no. gxyq2018038).

\section{Availability of data and materials}

Not applicable.

\section{Authors' contributions}

FS provided the case, formulated the therapeutic regimen and revised the manuscript. $\mathrm{ZW}$ and RW aided in patient management. YL collected the imaging data. TZ collected clinical data and wrote the manuscript. QW and SQ performed the analyses with constructive discussions. All the authors have read and approved the final version of this manuscript.

\section{Ethics approval and consent to participate}

Not applicable.

\section{Patient consent for publication}

Informed consent for publication was obtained from the family member of the patient.

\section{Competing interests}

The authors declare that they have no competing interests.

\section{References}

1. Silva JA, Mesquita Kde C, Igreja AC, Lucas IC, Freitas AF, Oliveira SM, Costa IM and Campbell IT: Paraneoplastic cutaneous manifestations: Concepts and updates. An Bras Dermatol 88: 9-22, 2013.

2. Sterz G: Polymyositis. Berliner Klinische Wochenschrift 53: 489, 1916.

3. Zhang $\mathrm{X}$, Wang $\mathrm{Y}$, Ma $\mathrm{G}$, Zhang L, Jing $\mathrm{H}$ and Du J: Dermatomyositis as a symptom of primary lung cancer: A case report and review of the literature. Oncol Lett 11:3413-3416, 2016.

4. de Souza FH and Shinjo SK: Newly diagnosed dermatomyositis in the elderly as predictor of malignancy. Rev Bras Reumatol 52: 713-721, 2012 (In English, Portuguese).

5. Zahr ZA and Baer AN: Malignancy in myositis. Curr Rheumatol Rep 13: 208-215, 2011.

6. Miret M, Horváth-Puhó E, Déruaz-Luyet A, Sørensen HT and Ehrenstein V: Potential paraneoplastic syndromes and selected autoimmune conditions in patients with non-small cell lung cancer and small cell lung cancer: A population-based cohort study. PLoS One 12: e0181564, 2017.

7. Porto L, Miranda M, Gomes A, André R and Rodrigues B: Paraneoplastic neurological syndrome as an initial indicator of small cell carcinoma of the lung. BMJ Case Rep 2013: bcr2012008432, 2013.

8. Santacroce L: Paraneoplastic syndromes. Drugs \& Diseases, 2017. https://emedicine.medscape.com/article/280744-overview. Updated December 06, 2018.

9. Kanaji N, Watanabe N, Kita N, Bandoh S, Tadokoro A, Ishii T, Dobashi $\mathrm{H}$ and Matsunaga T: Paraneoplastic syndromes associated with lung cancer. World J Clin Oncol 5: 197-223, 2014

10. Sparsa A, Liozon E, Herrmann F, Ly K, Lebrun V, Soria P, Loustaud-Ratti V, Bouyssou-Gauthier ML, Boulinguez $\mathrm{S}$, Bédane $\mathrm{C}$, et al: Routine vs extensive malignancy search for adult dermatomyositis and polymyositis: A study of 40 patients. Arch Dermatol 138: 885-890, 2002.

11. Basset-Seguin N, Roujeau JC, Gherardi R, Guillaume JC, Revuz J and Touraine R: Prognostic factors and predictive signs of malignancy in adult dermatomyositis. A study of 32 cases. Arch Dermatol 126: 633-637, 1990.

12. Pelosof LC and Gerber DE: Paraneoplastic syndromes: An approach to diagnosis and treatment. Mayo Clin Proc 85: 838-854, 2010.

13. Iorizzo LJ III and Jorizzo JL: The treatment and prognosis of dermatomyositis: An updated review. J Am Acad Dermatol 59: 99-112, 2008

14. Kovacs SO and Kovacs SC: Dermatomyositis. J Am Acad Dermatol 39: 899-920, 1998. 